\title{
Structural investigation of the (110) surface of $\gamma-\mathrm{Al}_{4} \mathrm{Cu}_{9}$
}

\author{
É. Gaudry, A. K. Shukla, T. Duguet, J. Ledieu, M.-C. deWeerd, J.-M. Dubois, and V. Fournée* \\ Institut Jean Lamour, UMR 7198 CNRS, Ecole des Mines, Nancy Université-UPV Metz, Parc de Saurupt, 54042 Nancy Cedex, France
}

(Received 25 May 2010; published 9 August 2010)

\begin{abstract}
We present a detailed study of the (110) surface of the $\gamma-\mathrm{Al}_{4} \mathrm{Cu}_{9}$ crystal using both experimental methods and first-principles calculations based on density-functional theory. Our experimental approach, using lowenergy electron diffraction, scanning tunneling microscopy (STM) images, and x-ray photoelectron spectroscopy highlights the presence of two types of surface terminations. Combining experimental results and simulations provides many arguments to match the two observed surfaces with the two puckered terminations built from bulk truncation: (i) calculations show that these two puckered terminations present lower surface energies compared to another conceivable flat termination obtained also from bulk truncation, (ii) step height measurements are consistent with calculated interlayer spacings and (iii) simulated STM images are in reasonable agreement with the experimental ones and mirror the experimental voltage dependence.
\end{abstract}

DOI: 10.1103/PhysRevB.82.085411

PACS number(s): 61.44.Br, 68.35.bd, 68.37.Ef

\section{INTRODUCTION}

Quasicrystals are intermetallic alloys with long-range aperiodic order exhibiting rotational symmetries that are incompatible with translational periodicity. ${ }^{1}$ Approximants are related crystalline phases with chemical composition close to that of the parent quasicrystalline phase. ${ }^{2}$ Their unit cell contains from several tens to a few thousands of atoms, typically arranged into highly symmetric clusters similar to those encountered in the parent quasicrystalline phases.

The surface of these materials have attracted a lot of interest in the past decade, driven by potentially useful chemical and mechanical properties. ${ }^{3,4}$ These include promising catalytic properties and good oxidation resistance ${ }^{5-9}$ as well as poor wetting and low-friction coefficients. ${ }^{3,10,11}$ A complete undestanding of these properties requires a detailed knowledge of the atomic arrangement at their surface.

Over the last fifteen years, experimental studies of clean quasicrystalline surfaces have been carried out using ultrahigh vacuum techniques such as low-energy electron diffraction (LEED) and scanning tunnelling miscroscopy (STM). ${ }^{12-17}$ It was concluded that the topmost surface of these complex metallic alloys could be understood as a simple truncation of the bulk structure with the particularity that only specific planes appear at the surface along a given crystallographic direction. More recently, a limited number of surface studies of periodic complex metallic alloys has also been reported and selection of specific planes as surface termination is also observed. This is the case for the pseudotenfold surface of the $\xi^{\prime}-\mathrm{Al}_{77.5} \mathrm{Pd}_{19} \mathrm{Mn}_{3.5}$ crystal, an approximant of the $i$-Al-Pd-Mn quasicrystal, ${ }^{18,19}$ the pseudotenfold surface of the $\mathrm{Al}_{13} \mathrm{Co}_{4}$ crystal, an approximant of the Al$\mathrm{Ni}$-Co quasicrystal, ${ }^{20}$ and the (010) surface of the orthorhombic $\mathrm{T}-\mathrm{Al}_{3}(\mathrm{Mn}, \mathrm{Pd})$ phase, an approximant of the stable decagonal Al-Pd-Mn quasicrystal. ${ }^{21}$

The driving force for the selection of specific planes as surface termination is related to the minimization of the surface free energy, defined as the excess free energy at the surface of a material compared to the bulk. In a simple model, the surface energy is proportional to the number of missing bonds at the surface. This broken-bond rule accounts for the greater stability of the densest planes in the case of simple metals. ${ }^{22,23}$ More generally, the surface-free-energy minimization leads to the emergence of preferred surface orientations and for a given $(h k l)$ surface, interlayers relaxations, and possible surface reconstructions. In alloys, the introduction of different chemical elements offers additional mechanisms to minimize the surface energy, through the selection of specific planes or chemical segregation at the surface. However, in the case of complex metallic alloys, experiments have shown that no segregation takes place. $^{13,14,24-27}$ Thus, the selection of specific atomic planes as topmost layers relies on parameters such as their atomic density, their chemical composition, and interlayer distances. ${ }^{28}$ In Al-based quasicrystals, given that the elemental surface energy of $\mathrm{Al}$ is generally lower than that of other constituents in Al-based quasicrystals (the surface energy is around 1.2-1.3 $\mathrm{J} \mathrm{m}^{-2}$ for $\mathrm{Al}$ and $1.9-2.1 \mathrm{~J} \mathrm{~m}^{-2}$ for $\mathrm{Pd}$ or $\mathrm{Mn}$, for example), ${ }^{29}$ one expects dense Al-rich planes to appear at the surface. Given furthermore that a large interplanar gap generally implies weaker bonding between planes and thus a lower surface energy, the selection rules should also take into account the interplanar spacing. This is consistent with experimental findings for Al-based icosahedral quasicrystals, for which the fivefold surfaces terminate preferentially in large spaces which are located just above the atomic layer of relatively large atomic density and high $\mathrm{Al}$ content. ${ }^{30}$ The above considerations should be also valid for approximant phases.

Here, we examine more quantitatively the validity of these simple rules by using experimental results combined with numerical simulations. We focus on the (110) surface of $\gamma-\mathrm{Al}_{4} \mathrm{Cu}_{9}$ a system of intermediate complexity compared to large approximants and quasicrystals. The $\gamma-\mathrm{Al}_{4} \mathrm{Cu}_{9}$ phase has a simple cubic structure with a lattice parameter equal to $8.71 \AA$ and $c P 52\left(D 8_{3}, P \overline{4} 3 m\right)$ space group. ${ }^{31,32}$ It is based on a $3 \times 3 \times 3 \quad \mathrm{CsCl}$ superstructure containing two vacancies. ${ }^{33}$ Alternatively, it can be described by a bcc packing of 26-atoms clusters. It is a Hume-Rothery phase stabilized by the Fermi sphere-Brillouin zone boundary interaction. ${ }^{33,34}$ It has a number of valence electrons per unit volume $\left(0.127 e^{-} \AA^{-3}\right)$ close to that of the icosahedral Al- 
$\mathrm{Cu}-\mathrm{Fe}$ phase $\left(0.124 e^{-} \AA^{-3}\right){ }^{33}$ Its atomic structure also shows some similarities with that of decagonal phases. In particular, pentagonal motifs can be found within the (110) atomic planes of $\gamma-\mathrm{Al}_{4} \mathrm{Cu}_{9}$ that form chains with pseudodecagonal symmetry extending along the [110] direction (see Fig. 6 in Sec. IV). In addition, the $\gamma-\mathrm{Al}_{4} \mathrm{Cu}_{9}$ phase can be described by the staking of two different kinds of $\{110\}$ atomic layers along the [110] direction. The latter suggests that $\mathrm{Al}_{4} \mathrm{Cu}_{9}$ can be used as a model system to investigate the mechanism of surface planes selection in complex metallic alloys. In addition, it can be formed as a surface alloy in orientational relationship on quasicrystalline substrates. ${ }^{35-37}$

The paper is organized as follows. Section II describes the single-crystal growth along with experimental and computational details. Sections III and IV summarize all experimental and computational results. Section V shows how the combined experimental and computational results lead to the determination of the surface atomic planes. In Sec VI, a parallel is drawn with previous observations of terrace selection at quasicrystalline surfaces.

\section{METHODOLOGY}

\section{A. Single-crystal growth}

The $\gamma-\mathrm{Al}_{4} \mathrm{Cu}_{9}$ incongruently melts and has a reported width of formation $\mathrm{Al}_{100-x} \mathrm{Cu}_{x}$ ranging from $x$ equal 65.7 to 69.4 at. $\%{ }^{38} \mathrm{~A}$ mother ingot of the $\gamma-\mathrm{Al}_{4} \mathrm{Cu}_{9}$ phase was first prepared by induction melting the pure elements (Al-99.9\%, $\mathrm{Cu}-99.99 \%$ ) under an argon atmosphere. Then, the ingot was placed in an alumina crucible sealed within a quartz tube under vacuum $\left(10^{-4} \mathrm{mbar}\right)$ and homogenized by annealing at $1323 \mathrm{~K}$ for $4 \mathrm{~h}$. Single grains were formed by slow cooling. The system was first cooled down to $1093 \mathrm{~K}$ at a rate of $0.05 \mathrm{~K} / \mathrm{min}$ and down to room temperature at a rate of 1 $\mathrm{K} / \mathrm{min}$. At the end of the heat treatment, the sample had a cylindrical shape with only three grains visible by optical microscopy. The sample was finally cut along the grain boundaries to produce three single crystals with centimetric size.

The structure of the grown crystals was checked by powder x-ray diffraction on a Bragg-Brentano x-ray diffractometer (Co $K \alpha$ radiation; $\lambda=0.178897 \mathrm{~nm}$ ). The lattice parameter deduced from XRD pattern was $8.717 \AA$ in agreement with reported values. The chemical composition of the crystals was $\mathrm{Al}_{32.5}-\mathrm{Cu}_{67.5}$ (at. \%) as determined by energy-dispersive $\mathrm{x}$-ray spectroscopy (EDX) using a JEOL scanning electron microscope operating at $30 \mathrm{kV}$. A single crystal was then oriented by Laue backscattering such as to expose a (110) surface. The surface was finally polished using diamond paste down to $1 / 4$ micron.

\section{B. Surface analysis in ultrahigh vacuum}

Experiments were performed in an ultrahigh vacuum multichamber system with a base pressure of $5 \times 10^{-11}$ mbar. The $\gamma-\mathrm{Al}_{4} \mathrm{Cu}_{9}$ (110) surface was prepared by sputter $\left(\mathrm{Ar}^{+}, 2 \mathrm{keV}\right)$ and anneal cycles to temperatures ranging from 773 to $973 \mathrm{~K}$ for $1-2 \mathrm{~h}$. The temperature of the sample was monitored using an infrared optical pyrometer with the emis- sivity set to 0.35 coupled with a K-type thermocouple. This preparation method resulted in a step-terrace surface morphology. The long-range order of the surface was probed by LEED while the local atomic structure was probed by STM performed at room temperature using an Omicron VT-STM. The composition in the near-surface region was determined by core-level x-ray photoemission spectroscopy (XPS) using a nonmonochromatized $\mathrm{Mg} K \alpha(1253.6 \mathrm{eV})$ radiation source and an Omicron EA 125 electron analyzer operated in constant analyzer energy mode. The energy resolution was 0.8 $\mathrm{eV}$ at a pass energy of $20 \mathrm{eV}$. The valence band was measured by ultraviolet photoemission spectroscopy (UPS) using a He I source $(21.2 \mathrm{eV})$ with an energy resolution of $0.1 \mathrm{eV}$ at a pass energy of $2 \mathrm{eV}$.

\section{Computational details}

Structural optimizations and electronic-structure calculations were performed within the density-functional theory (DFT) framework, using the plane-wave Vienna $a b$ initio simulation package (VASP). ${ }^{39,40}$ Our calculations employ the ultrasoft potentials ${ }^{41}$ in the PW91 generalized gradient approximation $^{42}$ for the effective interaction of the valence electrons with the atomic cores.

Although the present calculations are called $a b$ initio, there are convergence parameters linked to the numerical implementation of the density-functional theory framework. Two of them are the plane-wave energy cutoff $E_{c u t}$ and the density of $k$-points sampling the Brillouin zone. A set of test calculations on bulk $\gamma-\mathrm{Al}_{4} \mathrm{Cu}_{9}$ (52 atoms/cell) was performed to determine the values of $E_{c u t}(400 \mathrm{eV})$ and the size of the Monkhorst-Pack $k$-points mesh $(7 \times 7 \times 7)$ in order to achieve a targeted precision for the total energy smaller than $0.0002 \mathrm{eV} /$ atom. A similar $k$-grid mesh $(5 \times 7 \times 1)$ was used for calculations using slabs whereas the $k$-grid mesh was increased $(7 \times 9 \times 1)$ for calculation of the density of states (DOS). The optimization of the atomic coordinates and unitcell parameters was performed by minimization of the Hellmann-Feynman forces acting on the atoms via a conjugate gradient algorithm. Simulations on the (110) surfaces of $\gamma-\mathrm{Al}_{4} \mathrm{Cu}_{9}$ were achieved by building symmetric slabs containing either 9, 11, or 13 atomic layers for the three kinds of surface terminations as described in the following part (see Sec. IV) and a vacuum layer with a thickness larger than $14.5 \AA$. The surface lattice parameters were set in agreement with the lattice parameters of the bulk alloy. Atomic relaxation was performed by allowing displacement of all atoms within the slab except those lying in the central atomic layer (layer number 5,6 , or 7 , respectively, when numbered along the direction perpendicular to the slab).

\section{EXPERIMENTAL RESULTS}

\section{A. Low-energy electron diffraction}

A typical LEED pattern of the (110) surface of the $\gamma-\mathrm{Al}_{4} \mathrm{Cu}_{9}$ phase is shown in Fig. 1. It was recorded at a primary beam energy of $60 \mathrm{eV}$ after annealing the surface at $900 \mathrm{~K}$. The reciprocal space distances can be calibrated using the diffraction pattern of a clean $\mathrm{Cu}(111)$ surface. This al- 


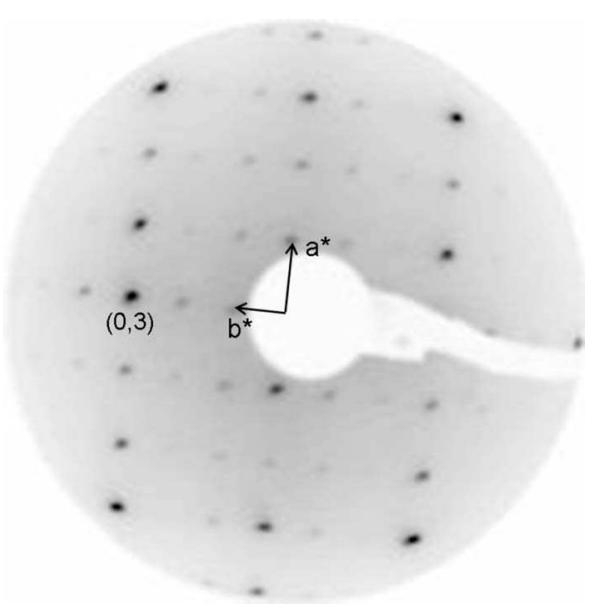

FIG. 1. LEED pattern of the $\gamma-\mathrm{Al}_{4} \mathrm{Cu}_{9}$ (110) surface recorded at a primary beam energy of $60 \mathrm{eV}$.

lows us to determine the dimensions of the $\gamma-\mathrm{Al}_{4} \mathrm{Cu}_{9}$ (110) surface unit cell which are $a=8.76 \pm 0.1 \AA$ and $b$ $=12.44 \pm 0.1 \AA$ with $b / a=1.42 \approx \sqrt{2}$. These values are in good agreement with surface lattice parameters expected from the bulk value $(a=8.717 \AA)$. No significant dependence of the spot intensities with the annealing temperature could be noticed, within the temperature range mentioned previously for the surface preparation. The most intense spots are the $(\mathrm{m}, 3 \mathrm{n})$ spots with $(\mathrm{m}, \mathrm{n})$ integers, but other spots have appreciable intensities as well. This is in contrast with earlier reports on the (110) surface of the $\gamma-\mathrm{Al}_{4} \mathrm{Cu}_{9}$ phase formed as surface alloys reported in Refs. 35 and 37 , where only $(\mathrm{m}, 3 \mathrm{n})$ spots had appreciable intensity. As mentioned earlier, the structure of the $\gamma-\mathrm{Al}_{4} \mathrm{Cu}_{9}$ phase can be described as a $3 \times 3 \times 3 \mathrm{CsCl}$ superstructure with two vacancies. ${ }^{33}$ The fact that all weak reflections were not observed in the LEED patterns was indicative of the presence of some disorder in such surface alloys, hindering the superstructure, which is not the case in the present study.

\section{B. Photoemission spectroscopy}

The chemical composition in the near-surface region can be obtained from XPS core-level analysis. It is estimated from the area of the $\mathrm{Cu} 3 s$ and $\mathrm{Al} 2 s$ core levels corrected by photoionization cross sections and photoelectron inelastic mean free path (see Fig. 2). A Shirley background has been used during peak fitting. The composition deduced from XPS spectra recorded at a take-off angle of $45^{\circ}$ is $\mathrm{Al}_{40 \pm 5} \mathrm{Cu}_{60 \pm 5}$, i.e., slightly enriched in $\mathrm{Al}$ compared to the composition determined by EDX but nevertheless close to the composition range of the $\gamma-\mathrm{Al}_{4} \mathrm{Cu}_{9}$ phase within experimental errors. Angle-dependent XPS measurements were used to vary the surface sensitivity of the analysis. The bottom panel in Fig. 2 shows the variation in the $\mathrm{Al}$ content as a function of the take-off angle of the photoelectrons defined as the angle between the surface normal and the analyzer. The surface sensitivity of the XPS signal increases with increasing take-off angle. More quantitatively, the information depth (ID) probed by XPS is defined as the sample thickness from
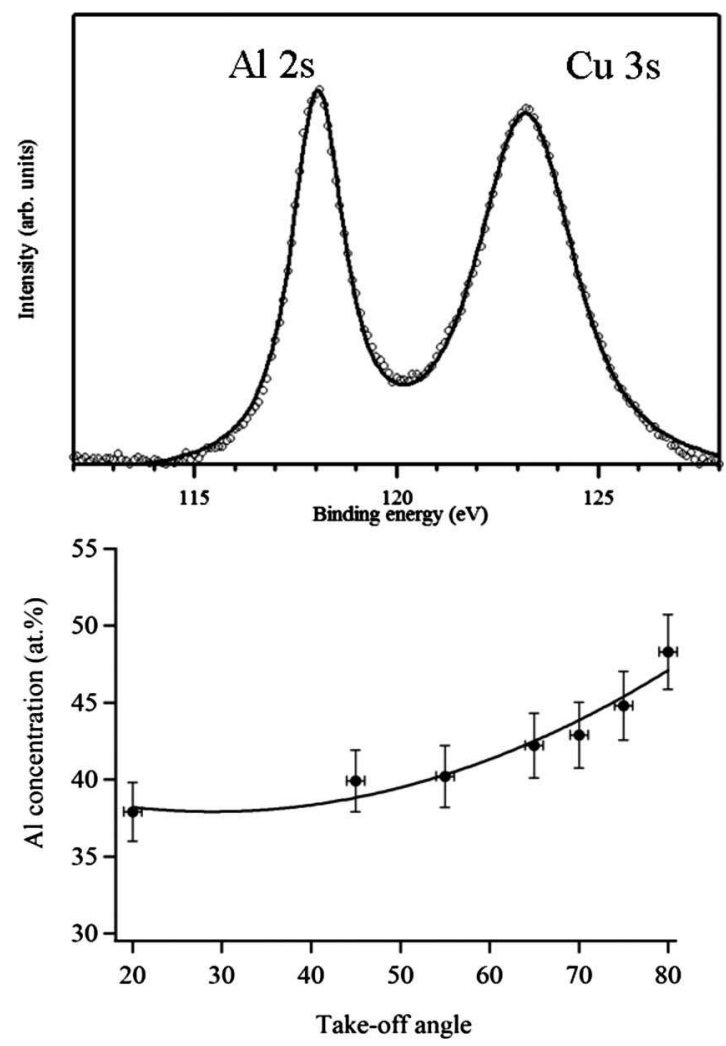

FIG. 2. Top: XPS spectra of the $\mathrm{Cu} 3 s$ and $\mathrm{Al} 2 s$ core levels of the $\gamma-\mathrm{Al}_{4} \mathrm{Cu}_{9}$ (110) surface recorded at a take-off angle of $45^{\circ}$. Bottom: calculated $\mathrm{Al}$ content (in at. \%) as a function of the take-off angle of the photoelectrons. Solid line is a fit of the experimental data points.

which a specified percentage $\mathrm{P}$ of the detected signal originates. ${ }^{43}$ Its analytical expression depends on the take-off angle $\phi: \mathrm{ID}=\lambda \cos \phi \ln [1-\mathrm{P} / 100]$, where $\lambda$ is the inelastic mean free path of the photoelectrons. The values of $\lambda$ for $\mathrm{Al} 2 s$ and $\mathrm{Cu} 3 s$ photoelectrons are similar as they have almost the same kinetic energy and are estimated at $26.8 \AA$ using the analytical formula proposed by Tanuma. ${ }^{44}$ The ID is thus reduced from $58 \AA$ at $\phi=20^{\circ}$ to $11 \AA$ at $\phi=80^{\circ}$ for $\mathrm{P}$ set to $90 \%$. Figure 2 shows an $\mathrm{Al}$ enrichment by about 10 at. $\%$ at the (110) surface of the $\gamma-\mathrm{Al}_{4} \mathrm{Cu}_{9}$ phase, suggesting that some chemical segregation takes place. Alternatively, such variations could also be explained by the anisotropy of the photoemitted signal from single crystals related to photoelectron diffraction effects. However, we have observed similar trends using a polycrystalline sample (not shown). The binding energies of the $\mathrm{Cu} 2 p_{3 / 2}, \mathrm{Cu} 3 s$, and $\mathrm{Al} 2 s$ in the $\gamma-\mathrm{Al}_{4} \mathrm{Cu}_{9}$ phase are $933.6 \mathrm{eV}, 123.3 \mathrm{eV}$, and $118.05 \mathrm{eV}$, respectively. Compared to the core levels measured on the pure metals using the same spectrometer, the $\mathrm{Cu}$ core levels are shifted by $0.95 \mathrm{eV}$ toward higher binding energies in the alloy. The chemical shift is much smaller for Al core levels $(+0.1 \mathrm{eV})$. Similar peak shifts have been reported for the $\gamma-\mathrm{Al}_{4} \mathrm{Cu}_{9}$ surface alloys as well as in other Al-Cu alloys. ${ }^{35,45}$

The valence band of the $\gamma-\mathrm{Al}_{4} \mathrm{Cu}_{9}$ phase has been recorded by UPS and is shown in Fig. 3. Due to photoionization cross-section effects, the spectra are dominated by the 

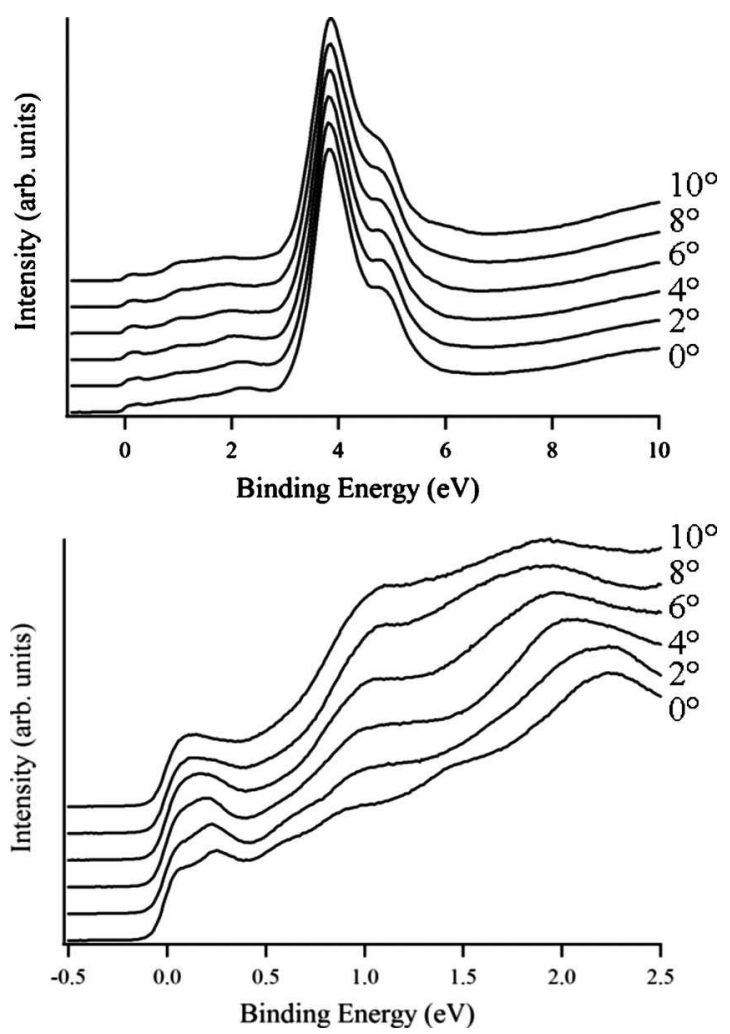

FIG. 3. Top: valence-band spectra of the $\gamma-\mathrm{Al}_{4} \mathrm{Cu}_{9}(110)$ surface recorded at different take-off angle. The main peak at $4 \mathrm{eV}$ corresponds to $\mathrm{Cu} d$ states. Bottom: near $E_{F}$ spectra showing dispersive features.

$\mathrm{Cu} d$ levels in the energy range between 3 and $6 \mathrm{eV}$ below $E_{F}$. The maximum of the $\mathrm{Cu} d$ band is located at $3.8 \mathrm{eV}$ binding energy and does not shift by varying the take-off angle away from normal emission. This value equals that reported for a polycrystalline $\gamma-\mathrm{Al}_{4} \mathrm{Cu}_{9}$ sample reported in Ref. 36. Dispersive features are observed in the energy range comprised between the $\mathrm{Cu} d$ band and the Fermi level, as seen in the bottom panel of Fig. 3. This is in agreement with calculated energy-dispersion relations for the $\gamma-\mathrm{Al}_{4} \mathrm{Cu}_{9}$ phase reported by Asahi et al.,${ }^{34}$ showing dispersive bands in the vicinity of the Fermi level and flat bands in the energy range of the $\mathrm{Cu} d$ band.

\section{Scanning tunneling microscopy}

The surface structure has been investigated by STM after annealing the sample between 773 and $973 \mathrm{~K}$. Within this temperature range, the surface has a step-terrace morphology but the step-height distribution varies. At low temperature $(773 \mathrm{~K})$, a single step height of $6.6 \pm 0.2 \AA$ is observed. Increasing the annealing temperature $(\geq 843 \mathrm{~K})$ results in step splitting into small $(\mathrm{S}=2.2 \pm 0.1 \AA)$ and large $(\mathrm{L}=4.4 \pm 0.1 \AA)$ steps. In the following, we call an $\mathrm{S}$ terrace (respectively, an L terrace) a terrace bounded by an S step downward (respectively, by an L step downward). We have measured the surface areas covered by the two different terraces from sets of STM images recorded after annealing the surface at different temperatures. We found that $\mathrm{S}$ and $\mathrm{L}$

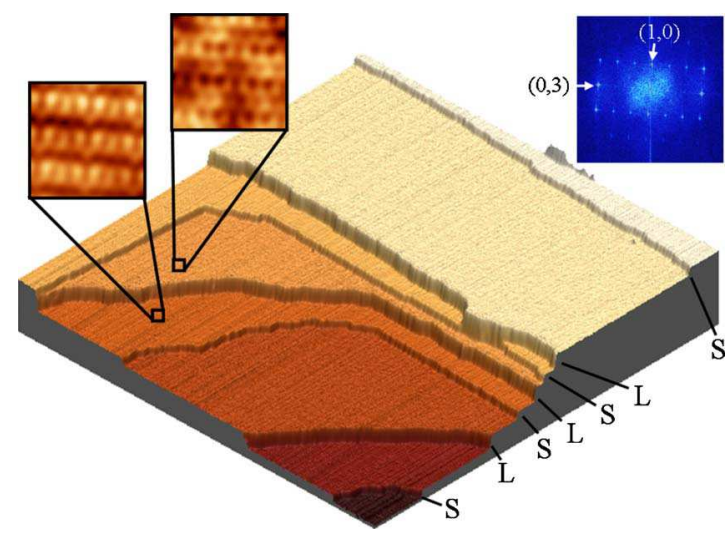

FIG. 4. (Color online) Three-dimensional view of the $\gamma-\mathrm{Al}_{4} \mathrm{Cu}_{9}$ (110) surface (STM, $200 \times 200 \mathrm{~nm}^{2}, I_{t}=0.2 \mathrm{nA}$, and $V_{b}=+1.4 \mathrm{~V}$ ) showing alternating $\mathrm{S}(2.2 \pm 0.1 \AA)$ and $\mathrm{L}(4.4 \pm 0.1 \AA)$ steps. Insets show the two different types of STM contrast observed on S and $\mathrm{L}$ terraces $\left(4 \times 4 \mathrm{~nm}^{2}, I_{t}=0.3 \mathrm{nA}\right.$, and $\left.V_{b}=+0.5 \mathrm{~V}\right)$ and the FFT of an STM image of $\gamma-\mathrm{Al}_{4} \mathrm{Cu}_{9}$ (110) surface.

terraces occupy equivalent surface areas within a temperature range of 843-903 $\mathrm{K}$ while $\mathrm{L}$ terraces become the preferred surface termination above $933 \mathrm{~K}$ covering about $80 \%$ of the total area.

The image shown in Fig. 4 has been obtained after annealing the sample at $933 \mathrm{~K}$, thus both types of terminations coexist at the surface. High-resolution STM images have been recorded on both $\mathrm{S}$ and $\mathrm{L}$ terraces revealing a different STM contrast as shown in the insets of Fig. 4. These two images have been extracted from an STM image spanning simultaneously both terraces with a bias of $+0.5 \mathrm{~V}$. The dimensions of the surface unit cell are easily determined from the fast Fourier transform (FFT) of STM images (see Fig. 4) and are $a=8.7 \pm 0.1 \AA$ and $b=12.4 \pm 0.1 \AA$, in perfect agreement with the dimensions determined by LEED. Note that $(0,3)$ spots are the most intense spots as it is the case in the LEED pattern. STM measurements are consistent with a bulk-terminated surface with no surface reconstruction. It further indicates that either one or two different (110) planes can be selected as surface terminations depending on the annealing temperature, suggesting that terminations corresponding to $\mathrm{L}$ terraces could be the most stable ones. Figure 5 shows $15 \times 15 \mathrm{~nm}^{2}$ STM images of L and S terraces recorded at a bias of $\pm 0.5 \mathrm{~V}$. The STM contrast shows important changes depending on whether the tunneling current is sampling the occupied or the unoccupied surface density of states. We will discuss this point later with the help of simulated STM images. Finally, we note that there is a relatively high density of defects at the surface. These defects are of two different types. The dark spots imaged as $2 \AA$ deep which can be seen in images shown in Fig. 5 (highlighted by arrows in top row images) are most likely surface vacancies. Other defects are possibly chemical inhomogeneities producing slight local variations in the STM contrast from one unit cell to the adjacent one. Such defects are highlighted in Fig. 5 by circles. They appear as patches of slightly darker contrasts at both negative and positive bias. These defects may originate from local chemical inhomogeneties at the nearsurface region, possibly related to an $\mathrm{Al}$ enrichment as mea- 


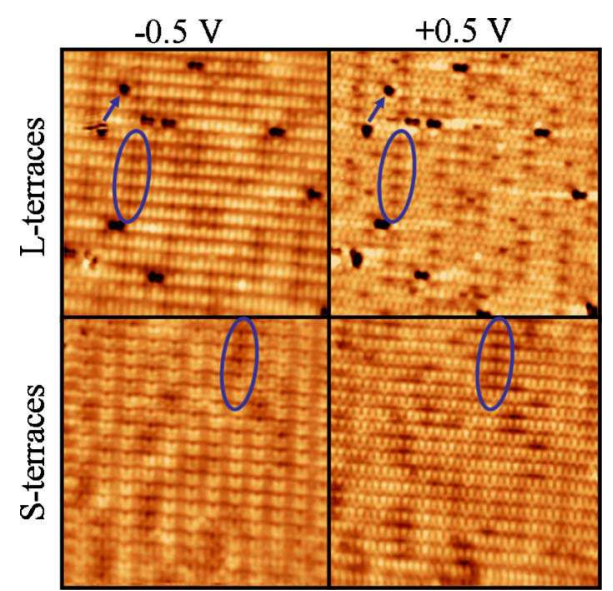

FIG. 5. (Color online) Images $\left(15 \times 15 \mathrm{~nm}^{2}, I_{t}=0.3 \mathrm{nA}\right)$ illustrating the bias dependency of the STM contrast for L and S terraces. Surface vacancies (arrows) and possible chemical inhomogeneities (circles) are highlighted.

sured by XPS. We stress that the magnitude of the Al enrichment is small enough to maintain the bulk truncated structure as observed by LEED and STM. We also note that the observed bias dependency of the STM contrast is an indication that the surface planes should contain $\mathrm{Cu}$ atoms.

Summarizing this experimental section, we have seen that LEED and STM data are consistent with a bulk-terminated surface in spite of some Al enrichment at the (110) surface of the $\gamma-\mathrm{Al}_{4} \mathrm{Cu}_{9}$ phase as measured by XPS. Two types of terminations can coexist depending on the surface preparation with one type ( $\mathrm{L}$ termination) being preferred over the other for annealing temperatures above $933 \mathrm{~K}$. In the following, the (110) surface of the $\gamma-\mathrm{Al}_{4} \mathrm{Cu}_{9}$ phase is investigated by numerical simulations.

\section{RESULTS FROM CALCULATIONS}

Optimization calculations of the bulk $\gamma-\mathrm{Al}_{4} \mathrm{Cu}_{9}$ leads to a lattice parameter of $8.75 \AA$, i.e., slightly larger than the measured bulk value of $8.717 \AA$. This is still in good agreement with experimental works as well as with previous calculations. ${ }^{31-33}$ For example, Mihalkovič and Widom ${ }^{46}$ reported a lattice parameter equal to $8.70 \AA$ Asing DFT calculations employing plane augmented waves pseudopotential whereas Cheng et al. ${ }^{47}$ obtained a value of $8.75 \AA$ using the embedded atom method. Total-energy calculations yield to a formation energy of the alloy equal to $\Delta H_{f}=2.92 \mathrm{eV}(0.225$ $\mathrm{eV} /$ atom), a value in good agreement with previous studies $(0.215$ eV/atom $) .{ }^{46}$

Figure 6 shows atomic structure of $\gamma-\mathrm{Al}_{4} \mathrm{Cu}_{9}$ projected along the [110] direction. Two kinds of atomic layers are stacked along this direction: flat atomic layers $F$ and $f(F$ type) and puckered atomic layers $\mathrm{P}, \mathrm{p}, \mathrm{p}^{\prime}$, and $\mathrm{P}^{\prime}$ ( $\mathrm{P}$ type). The $\mathrm{P}$ layers contain $12 \mathrm{Cu}$ atoms and six $\mathrm{Al}$ atoms per unit cell while $\mathrm{F}$ layers contain $12 \mathrm{Cu}$ atoms and four $\mathrm{Al}$ atoms per unit cell. From this model for bulk $\mathrm{Al}_{4} \mathrm{Cu}_{9}$, three different kinds of surface termination can be obtained by bulk truncation: a flat and two puckered terminations. It is worth mentioning that the flat atomic layers cut the 26-atoms clus-
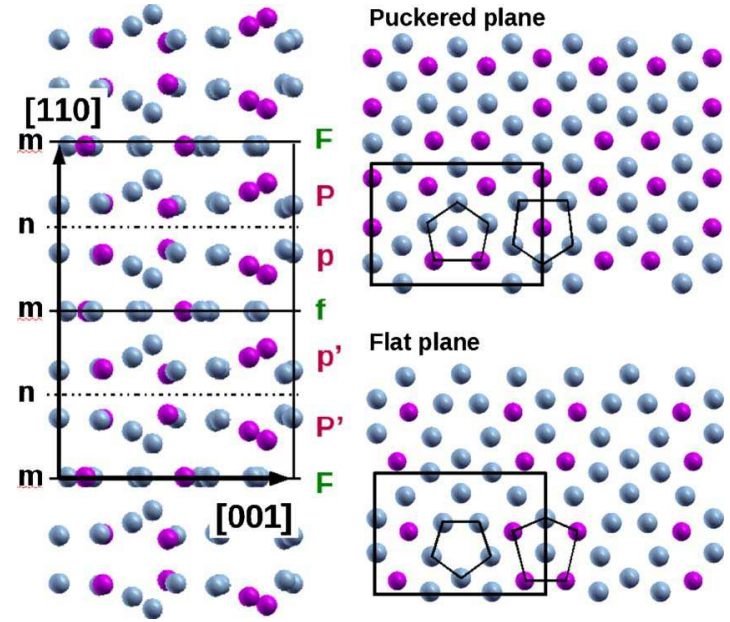

FIG. 6. (Color online) Left: atomic structure of $\gamma-\mathrm{Al}_{4} \mathrm{Cu}_{9}$ projected along the [110] direction. The letters $\mathrm{m}$ and $\mathrm{n}$ indicate the positions of mirrors planes. Right: atomic structures of puckered and flat planes. Light gray (blue) and dark gray (pink) dots are for $\mathrm{Cu}$ and $\mathrm{Al}$ atoms, respectively.

ters decorating the bcc lattice through their equatorial plane while both puckered atomic planes correspond to cut slightly above or below the equatorial plane. In the following, we will call PF the puckered surface termination that consists of a puckered layer that is above a flat layer and PP the puckered surface termination that is above a puckered layer. One could notice that in the PF termination (respectively, PP termination), the mean position of the aluminum atoms is slightly below (respectively, above) the mean position of the atomic plane.

\section{A. Energetics}

The energies of two surfaces having different stoichiometries cannot be compared directly by calculating the total energies of the two corresponding slabs. In order to analyze the relative surface stability of surfaces with different stoichiometries, we follow the methodology proposed in Refs. 48-50. The surface energy $\gamma_{\text {surf }}^{\alpha}$ of the termination $\alpha(\alpha=\mathrm{F}$, $\mathrm{PP}$, or PF) is evaluated from the total energy of a symmetric slab $E_{\text {slab }}^{\alpha}$ terminated by the $\alpha$ atomic layer by

$$
2 A \gamma_{\text {surf }}^{\alpha}=E_{\text {slab }}^{\alpha}-\sum_{i} \mu_{i} N_{i}
$$

where $A$ is the surface area and $\mu_{i}$ and $N_{i}$ are the chemical potential and the number of atom for each species $i$. It is possible to rewrite the previous equation with only one chemical potential, for example, $\mu_{\mathrm{Al}}$, using the relation expressing the thermal equilibrium between the surface and the bulk $\mathrm{Al}_{4} \mathrm{Cu}_{9}: \mu_{\mathrm{Al}_{4} \mathrm{Cu}_{9}}^{\text {bulk }}=4 \mu_{\mathrm{Al}}+9 \mu_{\mathrm{Cu}}$. Since $\mathrm{Al}$ and $\mathrm{Cu}$ elements form an alloy rather than segregate $\left(-\Delta H_{f}=\mu_{\mathrm{Al}_{4} \mathrm{Cu}_{9}}^{\text {bulk }}-4 \mu_{\mathrm{Al}}^{\text {bulk }}-9 \mu_{\mathrm{Cu}}^{\text {bulk }}<0\right)$, the chemical potential $\mu_{i}$ of the species $i$ in the alloy have to be smaller than the corresponding bulk chemical potential $\mu_{i}^{b u l k}$. These conditions give the range of variation for $\mu_{\mathrm{A}}$ 


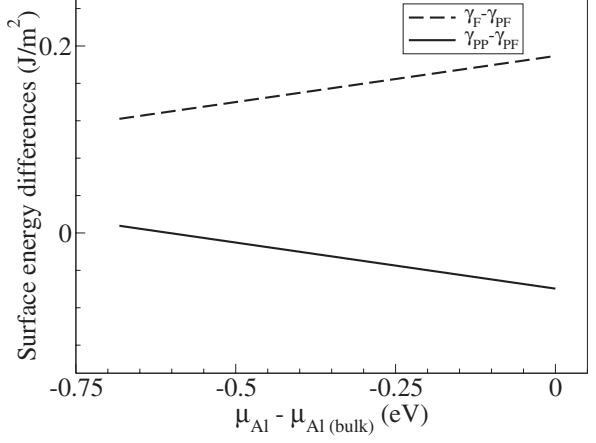

FIG. 7. Variation with the chemical potential of $\mathrm{Al}$ atoms of the surface energy of $\mathrm{F}$ and $\mathrm{PP}$ surface terminations referred to the PF termination.

$$
-\frac{\Delta H_{f}}{4}<\mu_{\mathrm{Al}}-\mu_{\mathrm{Al}}^{\text {bulk }}<0 .
$$

Finally, the expression for the surface energy becomes

$$
2 A \gamma_{\text {surf }}^{\alpha}=E_{\text {slab }}^{\alpha}-N_{\mathrm{Al}_{4} \mathrm{Cu}_{9}}^{\alpha} \mu_{\mathrm{Al}_{4} \mathrm{Cu}_{9}}^{b u l k}+N^{\alpha} \mu_{\mathrm{Al}},
$$

where $N_{\mathrm{Al}_{4} \mathrm{Cu}_{9}}^{\alpha}=\frac{N_{\mathrm{Cu}}}{9}$ and $N^{\alpha}=-N_{\mathrm{Al}}+\frac{4}{9} N_{\mathrm{Cu}}$.

The calculations described here have to be performed with slabs that are thick enough in order to avoid interaction between the two surfaces of the slab. However, a complete test of the convergence of the surface properties with the number of layers in the slab is computationally too demanding for such complex surfaces. For example, in the case of $\mathrm{PF}$ termination, such convergence test would require the comparison of the surface energies obtained with slabs built with $6 p+3$ atomic layers $(p \in \mathbb{N})$, leading to a number of atoms in the supercell equal to $104 p+52$. The corresponding calculations are possible with $p \leq 1$ but not for $p \geq 2$ because the number of atoms is already too large for such DFT calculations. However, convergence tests on more simple surfaces have been performed, leading to the conclusion that building slabs with seven or eight atomic layers is sufficient to provide well-converged surface energies. ${ }^{51}$ It is thus reasonable to use nine-layers, 11-layers, and 13-layers thick slabs to compare the surface energies of the PF, PP, and F terminations, respectively. We have also performed two calculations using either a seven-layers or a 13-layers thick symmetric slabs for the flat termination and found that the surface-energy difference is less than $10 \mathrm{~mJ} \mathrm{~m}^{-2}$, i.e., much smaller than the energy differences reported in Fig. 7.

Figure 7 show the surface-energy differences $\gamma_{\text {surf }}^{\text {flat }}-\gamma_{\text {surf }}^{\mathrm{PF}}$ and $\gamma_{\text {surf }}^{\mathrm{PP}}-\gamma_{\text {surf }}^{\mathrm{PF}}$ as a function of $\mu_{\mathrm{Al}}$. One clearly sees that the puckered terminations PF and PP are more stable than the flat one for the full range of allowed chemical potentials, the energy difference being larger than about $100 \mathrm{~mJ} \mathrm{~m}^{-2}$.

\section{B. Geometry}

The ideal (110) puckered terminated surfaces (PP and PF) obtained by bulk truncation shows negligible structural relaxations. The inward relaxation of the outermost PP surface plane is calculated to be less than $1 \%$ (the outward relaxation is of the same order of magnitude for the outermost PF sur-

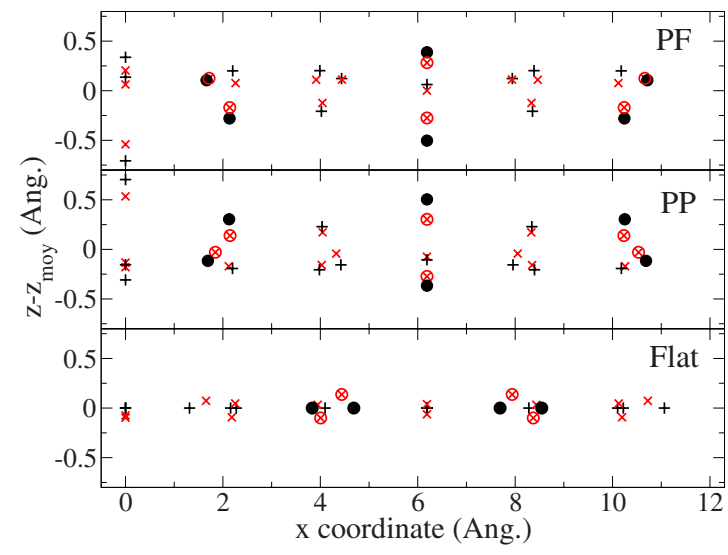

FIG. 8. (Color online) Atomic relaxation of the outermost atomic planes with respect to the corresponding bulk planes for the three types of terminations PP, PF, and $\mathrm{F}$ of the (110) $\gamma-\mathrm{Al}_{4} \mathrm{Cu}_{9}$ surface. The filled circles (respectively, plus features) are $\mathrm{Al}$ atoms (respectively, $\mathrm{Cu}$ atoms) in the bulk plane, the crossed circles (respectively, times features) are $\mathrm{Al}$ atoms (respectively, $\mathrm{Cu}$ atoms) in the surface planes.

face plane). The situation is quite different for the flat termination that shows a contraction of $4 \%$. These values are averaged values, since the three surface atomic planes are corrugated (see Fig. 8). In Fig. 8, one can notice that for both $\mathrm{PF}$ and PP surfaces, (i) the rippling of the puckered atomic planes is smaller for the surface planes compared to the corresponding bulk planes and (ii) the atomic $z$ displacement perpendicular to the surface is higher for $\mathrm{Al}$ or $\mathrm{Cu}$ atoms far from the mean position of the plane. The situation is slightly different in the case of the flat termination, where only aluminum atoms show a non-negligible displacement along the $z$ direction.

\section{STM images}

STM images are simulated within the Tersoff-Hamann approximation. ${ }^{52,53}$ In this scheme, the tunneling current $I$ is proportional to the local density of states at the position of the tip

$$
I(\mathbf{R})=\sum_{E_{n}>E_{F}-e V_{\text {bias }}}^{E_{n}<E_{F}}\left|\psi\left(\mathbf{R}, E_{n}\right)\right|^{2},
$$

where $\mathbf{R}$ is the position of the tip center of curvature, $E_{F}$ is the Fermi level, and $E_{n}$ the eigenstates of the crystal electrons. ${ }^{54}$ In most usual cases, the Tersoff-Hamann approximation provides sufficiently detailed STM simulations to account qualitatively for the experimental observations. ${ }^{55-58}$ The calculated images are shown in Fig. 9 for the three kinds of surface terminations discussed above.

In the case of the flat termination, no significant rippling is expected from calculations and the STM image obtained with $V_{\text {bias }}=+0.5 \mathrm{~V}$ mirrors roughly the positions of the atoms in the topmost plane. When comparing with the image obtained with $V_{\text {bias }}=-0.5 \mathrm{~V}$, one notices that the difference of contrast between the two images appears mainly around only copper atoms. In the case of the PP termination, the two 

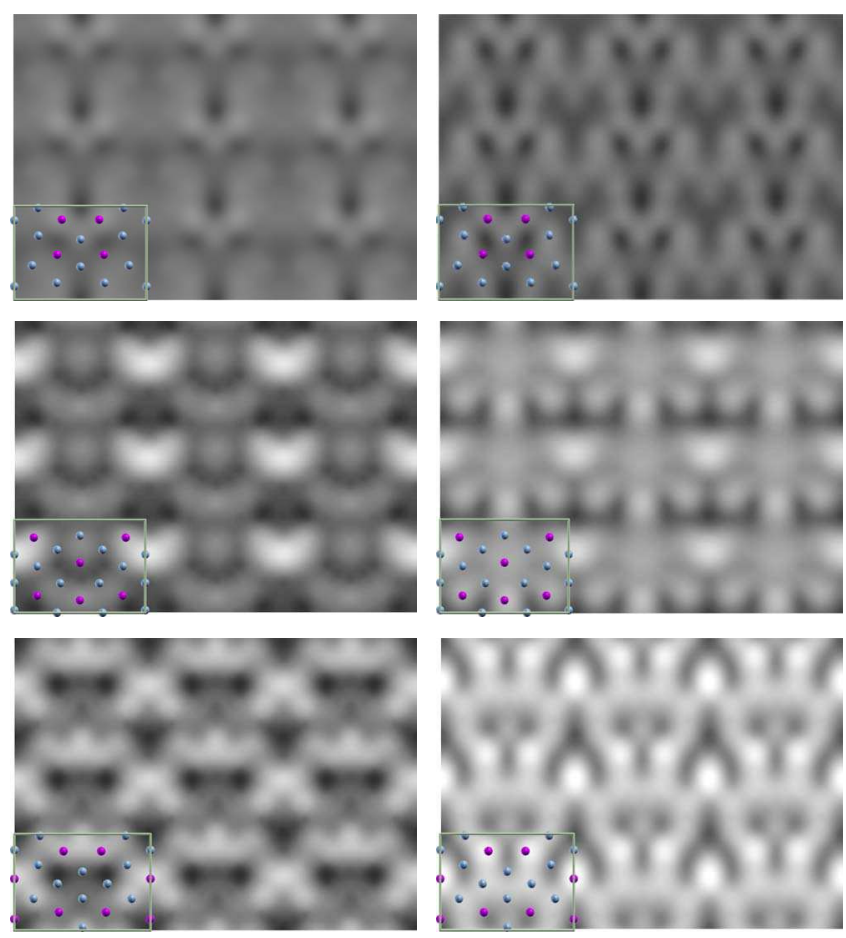

FIG. 9. (Color online) STM images simulated from the three bulk-terminated slabs: F (top), PP (middle), and PF (bottom). The images are plotted for a constant tip-surface distance $(\simeq 3 \AA)$ using two different bias voltages: $-0.5 \mathrm{~V}$ (left) and $+0.5 \mathrm{~V}$ (right). A unit cell is superimposed on the simulated images (the dark circles represent the aluminum atoms whereas the gray circles represent the copper atoms).

$\mathrm{Al}$ atoms that appear with bright features regardless of the sign of the applied bias voltage lie above the $z$ mean position of the topmost plane. Here, the constrast differences between the two images calculated with $V_{\text {bias }}= \pm 0.5 \mathrm{~V}$ appear around both copper and aluminum atoms. In the case of the $\mathrm{PF}$ termination, $\mathrm{Al}$ atoms have a bright contrast independently of the sign of the applied bias voltage and lie above the $z$ mean position of the topmost plane. Here, the contrast differences between the two images calculated with $V_{\text {bias }}= \pm 0.5 \mathrm{~V}$ appear mainly for copper atoms.

The STM simulation using the three structural models reproduce the bias dependence observed experimentally. The correspondence between the calculated and the experimental images is discussed in Sec. V A.

\section{Electronic structure of the puckered surfaces}

Figure 10 shows the calculated local DOS (LDOS) on surface and subsurface planes for the nine-layers thick $\gamma-\mathrm{Al}_{4} \mathrm{Cu}_{9}$ (110) slab with PF termination. We have also checked that similar type of results are obtained for the 11layers thick slab with PP termination. In Fig. 10, the label S refers to the surface plane, S-1 to the plane immediately below, etc.

When comparing the LDOS of the surface atomic plane to that of S-2 or S-3 layers, it is clear that the effect of the surface is mainly confined to the top surface layer. In the top

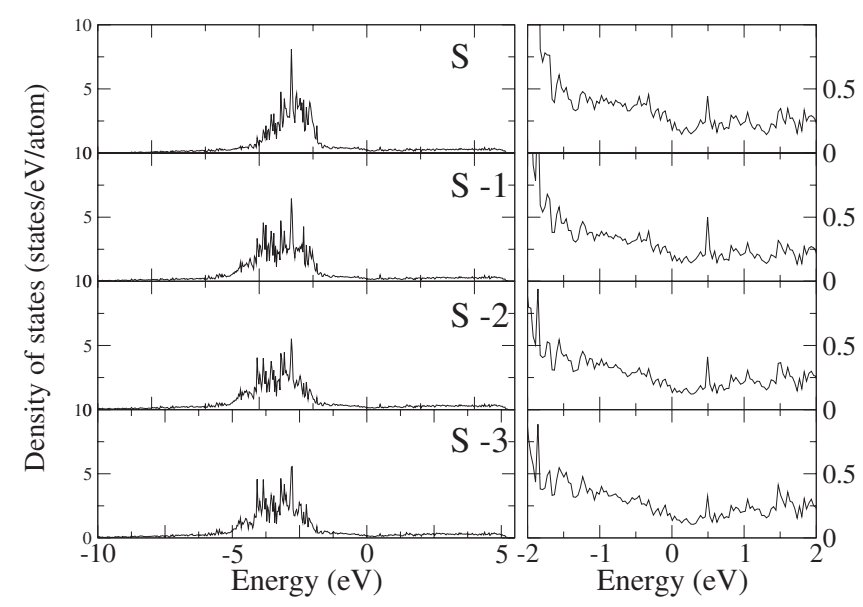

FIG. 10. Layer-decomposed local density of states calculated for a nine-layers thick slab with PF termination. The label $\mathrm{S}$ refers to the surface plane, S-1 is the subsurface plane, etc. Right panels are a blowup of the left ones in the vicinity of the Fermi level.

surface layer, the $\mathrm{Cu}-3 d$ bandwidth is reduced by about 0.4 $0.5 \mathrm{eV}$ compared to the bulk. The band narrowing is attributed to the reduction in the coordination number of surface atoms compared to the bulk. The $\mathrm{Cu} d$ band is also shifted by about $0.45 \mathrm{eV}$ toward lower binding energies compared to the "bulklike" S-3 Cu $d$ band, and the LDOS maximum is enhanced. A similar shift of the calculated transition-metal $d$-band center of mass of the surface LDOS has also been reported for the tenfold surface of the $d$-Al-Co-Ni quasicrystal $(\simeq 0.8 \mathrm{eV})$ and for the fivefold surface of $i$-Al-Pd-Mn quasicrystal $(\simeq 0.5 \mathrm{eV})$. These calculations were performed using structural models based on either $W$-(AlCoNi) or the 2/1-(AlPdMn) approximant phases. ${ }^{59,60}$

A worth noting feature on the density of states is the existence of a pseudogap at the Fermi level $E_{F}$ both in the DOS of the surface layer and in the DOS of the bulk material. This pseudogap is formed as a result of the resonance of electrons having nearly the Fermi energy with zones $\{330\}$ and $\{411\}$ and the formation of a set of standing waves separated in energy by a gap. ${ }^{34,37,61}$ The minimum of the pseudogap is slightly $(\simeq 0.25 \mathrm{eV})$ above $E_{F}$ for the bulklike S-3 layer; it is roughly at the same position for the surface layer and of the same order of magnitude (the surface DOS at $E_{F}$ increases by only percent compared to the bulklike $S-3$ layer). We note that the decrease in the depth of the pseudogap is much more pronounced in the case of the fivefold surface of $i$-Al-Pd-Mn quasicrystal (the surface DOS at $E_{F}$ increases by a factor of $5-6) .{ }^{62}$

\section{DISCUSSION}

\section{A. Combining experimental results and first-principles calculations}

At the (110) surface of the $\mathrm{Al}_{4} \mathrm{Cu}_{9}$ crystal, two different surface terminations ( $\mathrm{S}$ and $\mathrm{L}$ terraces) have been experimentally identified. The area covered by these $\mathrm{S}$ or L terminations largely depends on the surface preparation. The LEED patterns are consistent with a bulk-terminated surface within 

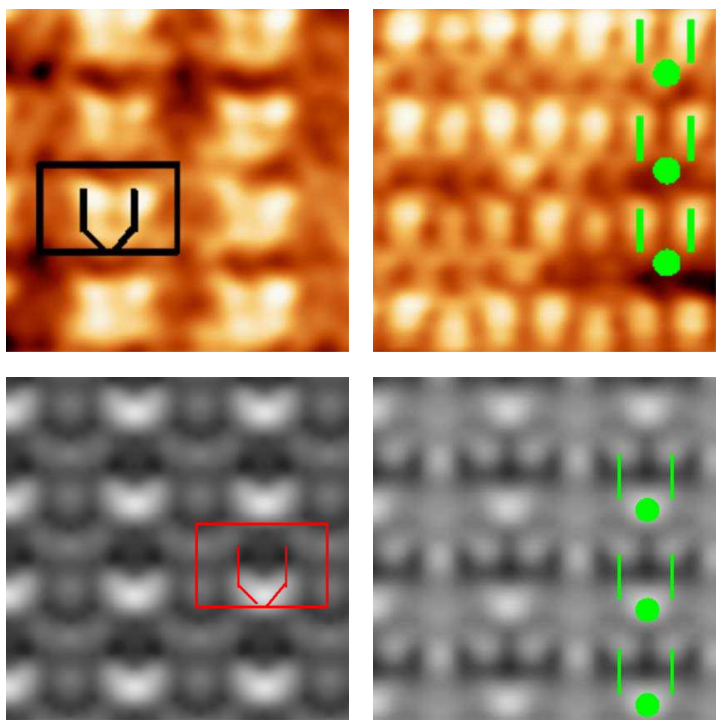

FIG. 11. (Color online) Comparison between experimental STM images recorded for the S-type terrace (top row) and simulated STM images for the PP model (bottom row). The left column corresponds to $V_{\text {bias }}=-0.5 \mathrm{~V}$, the right column to $V_{\text {bias }}=+0.5 \mathrm{~V}$.

the full range of annealing temperatures investigated. The surface lattice parameters correspond to those expected from the bulk. The surface composition measured by XPS falls within the stability range of the $\gamma-\mathrm{Al}_{4} \mathrm{Cu}_{9}$ phase. While not drastic, the angular dependence of the XPS signal indicates a slight $\mathrm{Al}$ enrichment at the surface. Step heights measurements indicate a sequence of small $(\mathrm{S}=2.2 \AA)$ and large $(\mathrm{L}=4.4 \AA)$ steps (LL or SS sequence are not observed). Because the interlayer spacings along the [110] direction are close to $2 \AA$, only two out of three possible bulk planes appear as surface termination. These step heights are consistent with values deduced from ab initio calculations for the $\cdots$-PF-PP-PF-PP- $\cdots$ periodic sequence $(S=2.18 \AA$ and $\mathrm{L}=4.02 \AA$ ) as well as for the $\cdots$-PF-F-PF-F- $\cdots$ sequence $(\mathrm{S}=2.03 \AA$ and $L=4.13 \AA)$. The alternative sequence $\cdots$-PP-F-PP-F- $\cdots$ seems unlikely since the calculated short distance is smaller $(\mathrm{S}=1.92 \AA)$.

Combining experimental and DFT results provides many arguments for the $\gamma-\mathrm{Al}_{4} \mathrm{Cu}_{9}$ (110) surface to be terminated at PF and PP planes. First, DFT calculations have shown that $\mathrm{PP}$ and PF terminations are more stable than the F termination. This result can be simply explained by the atomic surface density which is larger for puckered planes than that of flat planes. Indeed, the puckered layers contain two additional aluminum atoms per unit cell compared to flat layers. Furthermore, elemental surface energy of $\mathrm{Al}$ is lower than that of pure $\mathrm{Cu} \quad\left(\gamma_{\mathrm{Al}(111)}=0.75 \mathrm{~J} \mathrm{~m}^{-2}\right.$ and $\left.\gamma_{\mathrm{Cu}(111)}=1.41 \mathrm{~J} \mathrm{~m}^{-2}\right) .{ }^{51} \mathrm{In}$ addition, comparing experimental STM images recorded on $\mathrm{S}$ and $\mathrm{L}$ terraces at $V_{\text {bias }}= \pm 0.5 \mathrm{~V}$ with the corresponding simulated STM images, we find that the best match is obtained between $\mathrm{S}$ terraces and PP layers and L terraces and PF layers, respectively (see Figs. 11 and 12).

In addition, the experimental surface structure study shows that $\mathrm{L}$ terraces become the preferred surface terminations above $933 \mathrm{~K}$, covering about $80 \%$ of the total area.
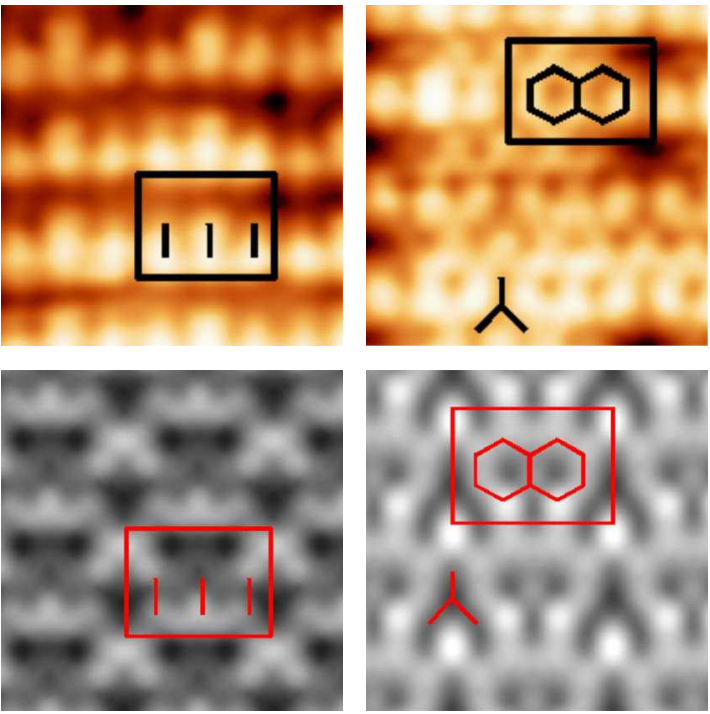

FIG. 12. (Color online) Comparison between experimental STM images recorded for the L-type terrace (top row) and simulated STM images for the PF model (bottom row). The left column corresponds to $V_{\text {bias }}=-0.5 \mathrm{~V}$, the right column to $V_{\text {bias }}=+0.5 \mathrm{~V}$.

\section{B. Electronic structure}

The electronic structure at the (110) surface of the $\gamma-\mathrm{Al}_{4} \mathrm{Cu}_{9}$ phase has been measured by scanning tunneling spectroscopy performed at room temperature. The ratio $\frac{d \ln I}{d \ln V}$ roughly reproduces the local density of states at the surface in the neighborhood of the Fermi level..$^{50}$

The bottom panel in Fig. 13 shows the experimental $\frac{d \ln I}{d \ln V}$ obtained by averaging over 2500 individual $I-V$ curves. The $\frac{d \ln I}{d \ln V}$ curve presents an asymmetric shape with a minimum located close to the Fermi level (the pseudogap) and two
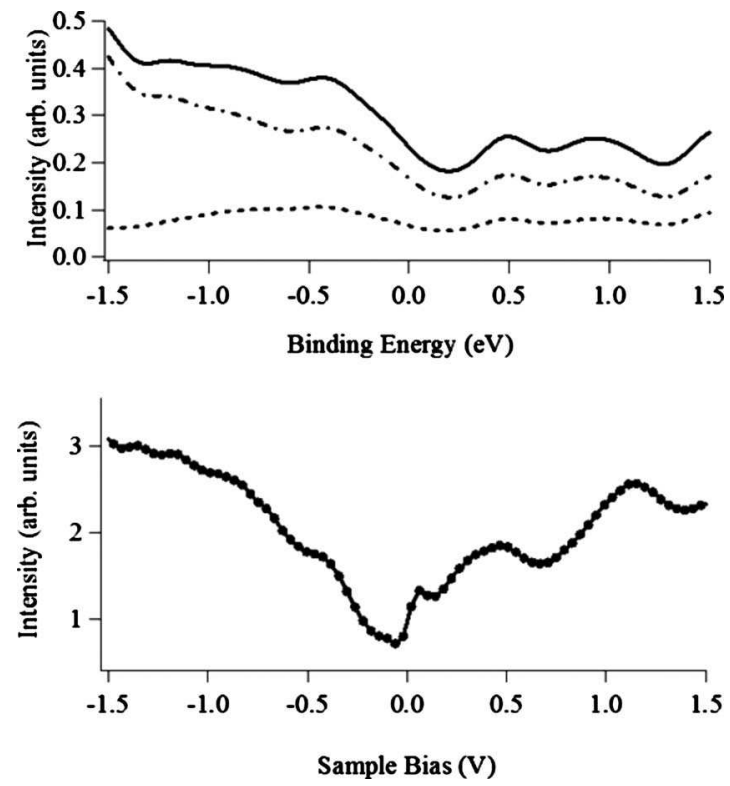

FIG. 13. Top: total DOS of the surface plane (solid line) and its decomposition into partial $\mathrm{Cu}$ (dotted-dashed line) and $\mathrm{Al}$ (dashed line) DOS. Bottom: experimental $\frac{d \ln I}{d \ln V}$ curve. 
local maxima at about 0.5 and $1.1 \mathrm{eV}$. These features are reproduced in the calculated surface density of states (DOS minimum located at about $0.23 \mathrm{eV}$ and two local maxima at 0.5 and $0.9 \mathrm{eV}$, see top panel of Fig. 13). When looking at the partial surface densities of states, it appears that the two maxima are mainly due to $\mathrm{Cu} p$ and $d$ states hybridized with $\mathrm{Al} p$ states. In addition, the decomposition of the density of states according to the positions of the atomic plane relative to the surface plane shows that these two maxima are still present in the bulklike S-3 atomic plane.

\section{Departure from the ideal picture}

In Sec. V A, we have have demonstrated that the $\mathrm{S}$ and $\mathrm{L}$ terraces can be understood by a bulk truncation at PP and PF planes. The interpretation of experimental images based on these two bulk planes are reasonable and in agreement with related experimental investigations of Al-bases cylindrical mirror analyzer (CMA) surfaces that point toward bulk truncated surfaces at specific planes characterized by a relatively high atomic density and an Al-rich content. ${ }^{13,14,24,27}$

However, the agreement between experimental and simulated STM images is not perfect, especially at negative bias. In addition, many defects are noticeable on the experimental STM images (see Fig. 5), manisfested by either $2 \AA$ dip or slight contrast variations. These defects may come from surface vacancies but also from Al surface segregation as suggested by XPS measurements. This is not too surprising as weak segregation effects have already been reported in $\alpha-\mathrm{Cu}-\mathrm{Al}$ solid solutions containing $9-16$ at. \% Al. ${ }^{63-66}$ This is in line with recent work ${ }^{67}$ performed by DFT on the segregation of $\mathrm{Cu}$ atoms embedded in an $\mathrm{Al}$ matrix showing that the presence of copper in the topmost layer is energetically unfavorable.

Segregation effects in the $\gamma-\mathrm{Al}_{4} \mathrm{Cu}_{9}$ compound and $\alpha$-Cu-Al solid solution cannot be compared directly. However, we have taken into consideration the following scenario where the Al concentration in the subsurface layers is the same as in the bulk while the topmost layer is composed of aluminum atoms only. We have tested this assumption by simulating the corresponding STM image and by comparing it with the experimental results. The calculations are performed for both P-type surface terminations. For the slab exhibiting the PF termination, Al segregation induces a change in chemical composition from $\mathrm{Al}_{30} \mathrm{Cu}_{70}$ for the bulk truncated model to $\mathrm{Al}_{44} \mathrm{Cu}_{56}$ in the $\mathrm{Al}$-segregated model (the composition is calculated by considering five atomic layers). For the slab with the PP termination, the composition of the bulk truncated model $\mathrm{Al}_{31} \mathrm{Cu}_{69}$ is shifted to $\mathrm{Al}_{42} \mathrm{Cu}_{58}$ in the Al-segregated model (here the composition is calculated by considering six atomic layers).

The simulated STM images are shown in Fig. 14. In the case of PP termination, the calculated images show lines of bright contrast that are not observed in experimental images. The agreement between simulated and experimental images is better for the PF termination. However the dark $Y$ features that clearly appear in both the experimental and simulated images using the bulk truncated model is not reproduced any more for the Al-segregated model. In addition, there is al-

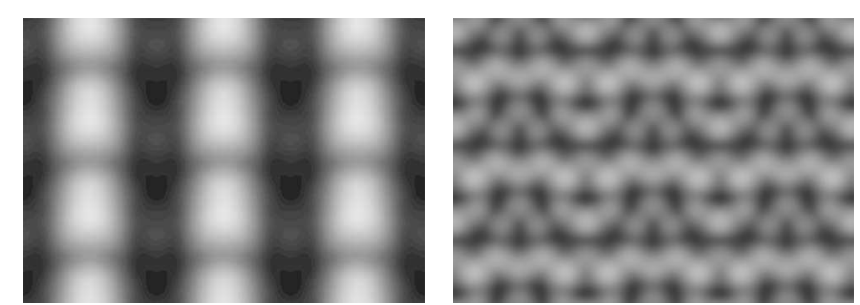

FIG. 14. Simulated images calculated from the P-type Alsegregated models described in the text. Left: PP topmost layer. Right: PF topmost layer.

most no bias dependency in the simulated images calculated using $V_{\text {bias }}= \pm 0.5 \mathrm{~V}$, in clear contradiction with experimental observation. The corresponding surface energies are also higher than those of the bulk truncated models (in the range $\left.\mu_{\mathrm{Al}}-\mu_{\mathrm{Al}}^{\text {bulk }}<-0.1 \mathrm{eV}\right)$, although the surface plane is pure Al. We can therefore conclude that the topmost layer cannot be a pure aluminum layer and must contain $\mathrm{Cu}$ atoms with a concentration that should be close to that of the corresponding bulk planes.

\section{CONCLUSION}

We have investigated the atomic and electronic structures of the (110) surface of the $\gamma-\mathrm{Al}_{4} \mathrm{Cu}_{9}$ phase by LEED, XPS, UPS, and STM combined with DFT calculations. Combining experimental results and first-principles calculations provide many arguments to identify the surface as a bulk truncation at specific planes. These planes are identified as puckered planes of the structural model. Flat planes characterized by a slightly lower atomic density do not appear as surface termination, in agreement with surface-energy calculations. This conclusion is in agreement with the general trends observed for related complex metallic alloy surfaces, although the degree of complexity of the $\gamma-\mathrm{Al}_{4} \mathrm{Cu}_{9}$ phase (measured by the number of atom per unit cell, for example) is sensibly lower: ${ }^{13,14,24-27}$ the densest Al-rich atomic planes (puckered planes), built from bulk truncation between two (110) atomic planes separated by the largest interlayer spacing, are selected as termination layers. However, many defects are noticeable on this surface compared to more complex CMA surfaces. Another difference is the weak-segregation effect that seems to take place on (110)- $\gamma-\mathrm{Al}_{4} \mathrm{Cu}_{9}$. The calculation of the energetic cost associated with the formation of a surface defect would be an interresting continuation of this work on CMA surfaces. A correlation could emerge between the defect energy and its position in the surface unit cell, i.e., with respect to the cluster units present in a particular CMA phase.

\section{ACKNOWLEDGMENTS}

The European Network of Excellence on "Complex Metallic Alloys," Contract No. NMP3-CT-2005-500145, and the Agence Nationale de la Recherche (Grant No. ANR-08BLAN-0041-01) are acknowledged for their financial support. 
*Corresponding author; vincent.fournee@mines.inpl-nancy.fr

${ }^{1}$ D. Shechtman, I. Blech, D. Gratias, and J. W. Cahn, Phys. Rev. Lett. 53, 1951 (1984).

${ }^{2}$ Basics of Thermodynamics and Phase Transitions in Complex Intermetallics edited by E. Belin-Ferré (World Scientific, Singapore, 2008).

${ }^{3}$ J.-M. Dubois, Useful Quasicrystals (World Scientific, Singapore, 2005).

${ }^{4}$ P. A. Thiel, Annu. Rev. Phys. Chem. 59, 129 (2008).

${ }^{5}$ C. Jenks and P. A. Thiel, J. Mol. Catal. A: Chem. 131, 301 (1998).

${ }^{6}$ A. P. Tsai and M. Yoshimura, Appl. Catal., A 214, 237 (2001).

${ }^{7}$ M. Yoshimura and A. P. Tsai, J. Alloys Compd. 342, 451 (2002).

${ }^{8}$ T. Tanabe, S. Kameoka, and A. P. Tsai, Catal. Today 111, 153 (2006).

${ }^{9}$ B. P. Ngoc, C. Geantet, M. Aouine, G. Bergeret, S. Raffy, and S. Marlin, Int. J. Hydrogen Energy 33, 1000 (2008).

${ }^{10}$ J.-M. Dubois, S. S. Kang, and J. V. Stebut, J. Mater. Sci. Lett. 10, 537 (1991)

${ }^{11}$ J. M. Dubois, P. Brunet, W. Costin, and A. Merstallinger, J. Non-Cryst. Solids 334-335, 475 (2004).

${ }^{12}$ M. Gierer, M. A. Van Hove, A. I. Goldman, Z. Shen, S.-L. Chang, C. J. Jenks, C.-M. Zhang, and P. A. Thiel, Phys. Rev. Lett. 78, 467 (1997).

${ }^{13}$ M. Gierer, M. A. Van Hove, A. I. Goldman, Z. Shen, S.-L. Chang, P. J. Pinhero, C. J. Jenks, J. W. Anderegg, C.-M. Zhang, and P. A. Thiel, Phys. Rev. B 57, 7628 (1998).

${ }^{14}$ Z. Papadopolos, G. Kasner, J. Ledieu, E. J. Cox, N. V. Richardson, Q. Chen, R. D. Diehl, T. A. Lograsso, A. R. Ross, and R. McGrath, Phys. Rev. B 66, 184207 (2002).

${ }^{15}$ Z. Papadopolos, P. Pleasants, G. Kasner, V. Fournée, C. J. Jenks, J. Ledieu, and R. McGrath, Phys. Rev. B 69, 224201 (2004).

${ }^{16}$ B. Unal, T. A. Lograsso, A. Ross, C. J. Jenks, and P. A. Thiel, Phys. Rev. B 71, 165411 (2005).

${ }^{17}$ H. R. Sharma, K. J. Franke, W. Theis, A. Riemann, S. Fölsch, P. Gille, and K. H. Rieder, Phys. Rev. B 70, 235409 (2004).

${ }^{18}$ V. Fournée, A. R. Ross, T. A. Lograsso, J. W. Anderegg, C. Dong, M. Kramer, I. R. Fisher, P. C. Canfield, and P. A. Thiel, Phys. Rev. B 66, 165423 (2002).

${ }^{19}$ H. R. Sharma, M. Shimoda, V. Fournée, A. R. Ross, T. A. Lograsso, and A. P. Tsai, Phys. Rev. B 71, 224201 (2005).

${ }^{20}$ R. Addou et al., Phys. Rev. B 80, 014203 (2009).

${ }^{21}$ T. Deniozou et al., Phys. Rev. B 81, 125418 (2010).

${ }^{22}$ I. Galanakis, G. Bihlmayer, V. Bellini, N. Papanikolaou, R. Zeller, S. Blügel, and P. H. Dederichs, Europhys. Lett. 58, 751 (2002).

${ }^{23}$ I. Galanakis, N. Papanikolaou, and P. H. Dederichs, Surf. Sci. 511, 1 (2002).

${ }^{24}$ T. Cai et al., Surf. Sci. 495, 19 (2001).

${ }^{25}$ L. Barbier, D. Le Floc'h, Y. Calvayrac, and D. Gratias, Phys. Rev. Lett. 88, 085506 (2002).

${ }^{26}$ J. Ledieu and R. McGrath, J. Phys.: Condens. Matter 15, S3113 (2003).

${ }^{27}$ R. D. Diehl, J. Ledieu, N. Ferralis, A. W. Szmodis, and R. McGrath, J. Phys.: Condens. Matter 15, R63 (2003).

${ }^{28}$ B. Unal, C. J. Jenks, and P. A. Thiel, Phys. Rev. B 77, 195419
(2008).

${ }^{29}$ H. L. Skriver and N. M. Rosengaard, Phys. Rev. B 46, 7157 (1992).

${ }^{30}$ M. Shimoda, H. Sharma, and A.-P. Tsai, Surf. Sci. 598, 88 (2005).

${ }^{31}$ L. Arnberg and S. Westman, Acta Crystallogr., Sect. A: Cryst. Phys., Diffr., Theor. Gen. Crystallogr. 34, 399 (1978).

${ }^{32}$ P. Villars and L. D. Calvert, Pearson's Handbook of Crystallographic Data for Intermetallic Phases (ASM International, Metals Park, Ohio, 1998).

${ }^{33}$ C. Dong, Philos. Mag. A 73, 1519 (1996).

${ }^{34}$ R. Asahi, H. Sato, T. Takeuchi, and U. Mizutani, Phys. Rev. B 71, 165103 (2005).

${ }^{35}$ M. Bielmann, A. Barranco, P. Ruffieux, O. Groning, R. Fasel, R. Widmer, and P. Groning, Adv. Eng. Mater. 7, 392 (2005).

${ }^{36}$ T. Duguet, J. Ledieu, J. M. Dubois, and V. Fournée, J. Phys.: Condens. Matter 20, 314009 (2008).

${ }^{37}$ T. Duguet, E. Gaudry, T. Deniozou, J. Ledieu, M. C. de Weerd, T. Belmonte, J. M. Dubois, and V. Fournée, Phys. Rev. B 80, 205412 (2009).

${ }^{38} \mathrm{http}: / /$ www.crct.polymtl.ca/FACT/documentation/SGTE/, 2004.

${ }^{39}$ G. Kresse and J. Furthmüller, Phys. Rev. B 54, 11169 (1996).

${ }^{40} \mathrm{G}$. Kresse and J. Furthmüller, Comput. Mater. Sci. 6, 15 (1996).

${ }^{41}$ D. Vanderbilt, Phys. Rev. B 41, 7892 (1990).

${ }^{42}$ J. P. Perdew and Y. Wang, Phys. Rev. B 45, 13244 (1992).

${ }^{43}$ C. J. Powell and A. Jablonski, Nucl. Instrum. Methods Phys. Res. A 601, 54 (2009).

${ }^{44}$ S. Tanuma, C. J. Powell, and D. R. Penn, Surf. Interface Anal. 21, 165 (1994).

${ }^{45}$ J. C. Fuggle, E. Kallne, L. M. Watson, and D. J. Fabian, Phys. Rev. B 16, 750 (1977).

${ }^{46}$ M. Mihalkovič and M. Widom, http://alloy.phys.phys.cmu.edu

${ }^{47}$ Y. Q. Cheng, E. Ma, and H. W. Sheng, Phys. Rev. Lett. 102, 245501 (2009).

${ }^{48}$ N. Moll, A. Kley, E. Pehlke, and M. Scheffler, Phys. Rev. B 54, 8844 (1996).

${ }^{49}$ L. Dias, A. Leitao, C. Achete, R.-P. Blum, H. Niehus, and R. Capaz, Surf. Sci. 601, 5540 (2007).

${ }^{50}$ F. Bechstedt, Principles of Surface Physics (Springer, Berlin, 2003).

${ }^{51}$ J. L. F. Da Silva, C. Stampfl, and M. Scheffler, Surf. Sci. 600, 703 (2006)

${ }^{52}$ J. Tersoff and D. R. Hamann, Phys. Rev. B 31, 805 (1985).

${ }^{53}$ J. Tersoff and D. R. Hamann, Phys. Rev. Lett. 50, 1998 (1983).

${ }^{54}$ A. Foster and W. Hofer, Scanning Probe Microscopy (Springer, Berlin, 2006).

${ }^{55}$ W. Hofer, Prog. Surf. Sci. 71, 147 (2003).

${ }^{56}$ J. Lauritsen and F. Besenbacher, Adv. Catal. 50, 97 (2006).

${ }^{57}$ J. Ledieu, M. Krajčí, J. Hafner, L. Leung, L. H. Wearing, R. McGrath, T. A. Lograsso, D. Wu, and V. Fournée, Phys. Rev. B 79, 165430 (2009).

${ }^{58}$ M. Krajčí, J. Hafner, J. Ledieu, and R. McGrath, Phys. Rev. B 73, 024202 (2006).

${ }^{59}$ M. Krajčí, J. Hafner, and M. Mihalkovič, Phys. Rev. B 73, 134203 (2006).

${ }^{60}$ M. Krajčí and J. Hafner, Phys. Rev. B 71, 054202 (2005).

${ }^{61}$ V. Fournée, I. Mazin, D. A. Papaconstantopoulos, and E. BelinFerré, Philos. Mag. B 79, 205 (1999). 
${ }^{62}$ M. Krajčí and J. Hafner, Phys. Rev. B 80, 214419 (2009).

${ }^{63}$ L. Zhu, E. Z. Muhlen, D. J. O'Connor, B. V. King, and R. J. MacDonald, Surf. Sci. 359, 54 (1996).

${ }^{64}$ R. J. Baird and T. J. Potter, J. Vac. Sci. Technol. A 3, 1371 (1985).
${ }^{65}$ Y. Yu, K. Sagisakaa, and D. Fujitaa, Surf. Sci. 603, 723 (2009). ${ }^{66}$ T. Kravchuk and A. Hoffman, Surf. Sci. 601, 87 (2007).

${ }^{67}$ A. Benali, Symposium on Surface Science, Austria, 2010 (unpublished). 